\title{
Cut-Off Scores of the Children's Depression Inventory for Screening and Rating Severity in Korean Adolescents
}

\author{
Young Rong Bang, Jae Hong Park ${ }^{\bowtie}$, and Sung Hwan Kim \\ Department of Psychiatry, Dong-A University College of Medicine, Busan, Republic of Korea
}

\begin{abstract}
Objective The aim was to establish an optimal cut-off score of the Children's Depression Inventory (CDI) for detecting depression and rating severity in Korean adolescents.

Methods A total of 468 students aged 12-16 years from 8 middle schools in Busan, Korea participated in this study. The Korean version of the CDI and Kiddie Schedule for Affective Disorders and Schizophrenia Present and Lifetime Version were used to evaluate depressive symptoms. Receiver-operating characteristics (ROC) analyses were conducted to evaluate case-finding performance and set the cut-off scores.

Results ROC analyses demonstrated that the overall discrimination power of the CDI is good enough to evaluate adolescent depression. The CDI sum score of 20 was identified as the optimal screening cut-off score, where sensitivity was 0.83 and specificity was 0.89 . This cut-off score could apply regardless of subjects' gender. The cut-off scores were examined in order of the depression severity: 15 for mild, 20 for moderate, and 25 for severe depression with high sensitivity and specificity.

Conclusion The CDI cut-off scores of our study can be recommended for screening depressed youth and rating the severity of depressive symptoms. The high negative predictive value suggested that the cut-off score of 20 would result in a small number of missed cases. Further studies are needed to ascertain these CDI cut-off scores for different age groups. Psychiatry Investig 2015;12(1):23-28
\end{abstract}

Key Words Children’s Depression Inventory, Cut-off score, Adolescent, Depression, Screening, Severity.

\section{INTRODUCTION}

Depression is a common mental health problem in adolescents, with an estimated lifetime prevalence of $17 \%{ }^{1}$ It has been well-known that depression has an upsurge throughout adolescence. ${ }^{2}$ Depression in adolescence predicts high degree of comorbidity and poor long-term outcome. Two-thirds of adolescents with depression have at least one comorbid psychiatric disorder such an anxiety disorder, ADHD, conduct disorder, and substance use disorder. ${ }^{3}$ Depressed youth are also at increased risk for educational underachievement, interpersonal problems, and suicidal behavior. Another major concern in adolescent depression is a high recurrence rate of de-

Received: November 22, 2013 Revised: February 26, 2014

Accepted: April 11, 2014 Available online: January 12, 2015

$\triangle$ Correspondence: Jae Hong Park, MD, PhD

Department of Psychiatry, Dong-A University College of Medicine, 26 Daesingongwon-ro, Seo-gu, Busan 602-715, Republic of Korea

Tel: +82-51-240-5467, Fax: +82-51-253-3542, E-mail: drpark@dau.ac.kr

(a) This is an Open Access article distributed under the terms of the Creative Commons Attribution Non-Commercial License (http://creativecommons.org/licenses/by$\mathrm{nc} / 3.0$ ) which permits unrestricted non-commercial use, distribution, and reproduction in any medium, provided the original work is properly cited. pression. Longitudinal studies have shown that the risk for recurrent depression in adolescent depression is extremely high, with the rate of recurrence ranging between $30-70 \%$ in the 1-2 years of follow-up in clinical samples, and $16-33 \%$ in the 2-3 years follow-up in community samples. ${ }^{4-6}$ Considering the high prevalence and adverse consequences associated with adolescent depression, it is of special interest to screen for depressed youth. For these reasons, several screening instruments have been used to detect depression early.

The Children's Depression Inventory (CDI) is one of the most widely used instruments for assessing the presence and severity of depressive symptoms in children and adolescents (age 7-17). ${ }^{7}$ The CDI can be completed by adolescents in approximately five to ten minutes and does not require specific training for scoring or interpretation. The CDI has established good reliability and validity for describing depressive symptoms, ${ }^{7,8}$ has good correlation with other scales, ${ }^{9}$ and high prediction validity for suicidal ideas. ${ }^{10}$ Thus the CDI has been used as a quick screening tool for depressive symptoms in clinical and general populations.

A cut-off score for the CDI was developed to differentiate 
between youth with and without a depressive disorder for the purposes of early detection and determining adequate treatments. Kovacs ${ }^{11}$ recommended that the cut-off score in clinical settings needs to be sensitive and is set at 13 . Meanwhile, in nonclinical samples, the cut-off score is 19 or 20, which seems to be specific enough to identify samples of potentially depressed children and adolescents. Many studies tested a cut-off score for their own countries, after considering the cultural and ethnic influences.

In developing the Korean version of the CDI, Cho and Lee ${ }^{12}$ postulated that the cut-off scores to detect depression in Korean population might be different from those used in Western countries because of cultural difference. However, there has been no published study yet to propose a cut-off score supported by systematic research. Therefore, although many clinicians and researchers have applied the CDI in their practice or research, there is still ambiguity about the diagnostic threshold of depression. ${ }^{13,14}$

Taking this into consideration, the present study had two major aims: 1) to establish an optimal cut-off score for the CDI when detecting depression in Korean adolescents; 2) to identify cut-off scores which classify severity of depression.

\section{METHODS}

\section{Participants and procedures}

The sample consisted of students aged 12-16 years from 8 middle schools in Busan, Korea. School recruitment consisted of contacting individual school principals to obtain permission to contact students for study participation. All students participated in the study after informed consent was obtained. Informed consent was attained from 649 students and their legal guardians. Students were requested to complete the CDI before diagnostic interview. The individual diagnostic interviews were carried out using the Korean version of the Kiddie Schedule for Affective Disorders and Schizophrenia Present and Lifetime Version (K-SADS-PL-K). A total of 54 (8.3\%) of students gave incomplete responses on the CDI and their questionnaires were excluded from the analysis. An additional 109 (16.8\%) of respondents did not participate in the diagnostic interview and were also excluded from the analysis. We were thus able to analyze the data from 468 (72.1\%) of students. Ethical approval for the study was obtained from the Institutional Review Board of Dong-A University Hospital.

\section{Measures}

The Korean version of the CDI and K-SADS-PL-K were used to evaluate depressive symptoms and severity. The Clinical Global Impression-Severity (CGI-S) was administered as an adjunctive measure to quantify severity of depressive symptoms.

\section{Children's depression inventory (CDI)}

There are 27 items quantifying symptoms such as depressed mood, hedonic capacity, vegetative functions, self-evaluation, and interpersonal behaviors. Each item consists of three statements graded in order of increasing severity from 0 to 2; children and adolescents select the one that characterized their symptoms best during the past 2 weeks. The item scores are combined into a total depression score, which ranges from 0 to 54. A higher CDI score means a higher depressive state. We used a Korean version of the $\mathrm{CDI}^{12}$ which demonstrated good reliability and validity for assessing depressive symptoms.

\section{Korean version of the kiddie schedule for affective} disorders and schizophrenia present and lifetime version

In the present study, K-SADS-PL-K was used as a gold standard measure to confirm diagnosis and rate severity of major depression according to DSM-IV-TR ${ }^{15}$ criteria. The K-SADSPL-K has good reliability and validity when diagnosing psychiatric disorders in children and adolescents. ${ }^{16}$ The K-SADSPL-K was administered by four professional psychiatrists, six psychiatry residents, and six clinical psychologists who were blinded to each participant's scores on the CDI. After two training sessions and three case rehearsals, inter-rater agreement for psychiatric diagnosis was substantial $(\kappa=0.75)$.

\section{Clinical global impression-severity}

The CGI-S rates the severity of the patient's illness, on a 7-point scale ranging from 1 (normal) to 7 (extremely Ill), according to the clinician's experience of patients suffering from the same condition. ${ }^{17}$ Higher scores therefore signify greater severity and/or worse outcomes.

In our study, we used the CGI-S as an auxiliary tool to rate symptom severity and K-SADS-PL-K as a gold standard. DSMIV-TR provides guidelines but not quantifiable methods to rate severity of depression according to intensity of symptoms and social functioning impairment. Thus, we applied the CGI$\mathrm{S}$ as a quantifiable measure to rate symptom severity. At the outset, participants were categorized into three groups according to severity (i.e., mild, moderate, and severe) after K-SADSPL-K interview. Next, we compared the mean CGI-S scores of the three severity groups. It was assumed that the correspondence of the mean CGI-S scores and the severity groups would confer additional validity to the severity classification based on the K-SADS-PL-K interview.

\section{Statistical analysis}

We conducted receiver-operating characteristics (ROC) analyses for CDI sum scores to evaluate case-finding performance. Diagnosis of major depressive disorder was assigned according to the K-SADS-PL-K which served as an external 
criterion. The accuracy of the CDI in detecting depression was measured by the area under the ROC curve (AUC). AUC values of $\geq 0.90$ are considered excellent, $0.80-0.90$ good, $0.70-$ 0.80 fair, and $<0.70$ poor. ${ }^{18}$ ROC analyses were performed for the total subjects and subgroups based on gender.

We calculated the sensitivity, specificity, positive predictive value (PPV) and negative predictive value (NPV) for candidate cut-off points. Optimal cut-off values were those that demonstrated a maximal Youden Index $(Y)^{19}$ which represents the highest combination of sensitivity and specificity. McNemar's tests were used to calculate the differences in sensitivity and specificity.

To examine the severity cut-off scores, three ROC analyses were conducted. Patient groups with major depression were divided into mildly, moderately, and severely depressed patients. Mildly depressed patients were compared to controls, moderately depressed patients were compared to controls and

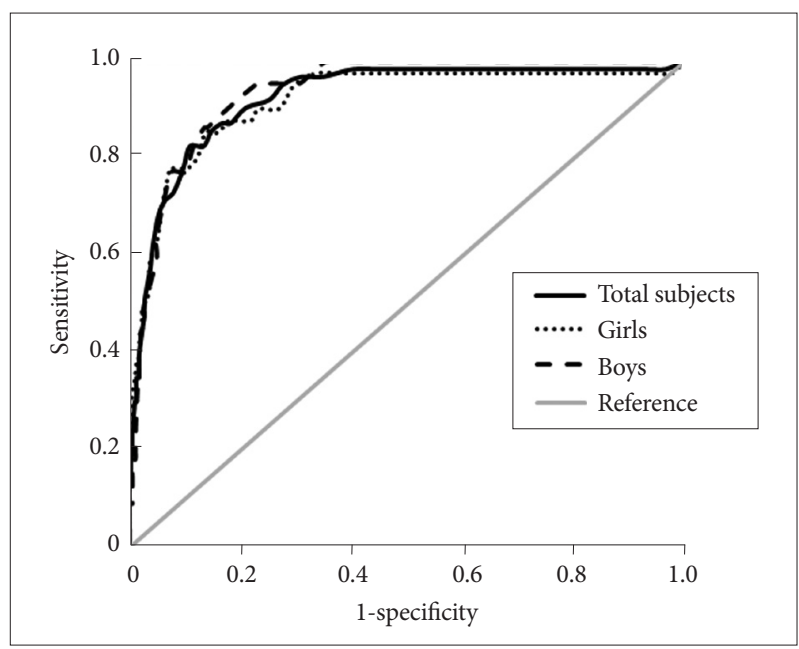

Figure 1. ROC curves of CDI sum scores for the complete samples and separated by gender. ROC: receiver-operating characteristics, CDI: Children's Depression Inventory. mildly depressed patients, and severely depressed patients were compared to controls and mildly and moderately depressed patients. One-way analysis of variance was carried out to compare the CGI-S scores of severity classification. All analyses were conducted using IBM SPSS Statistics 18.0 (version 18.0, SPSS Inc., Chicago, IL, USA).

\section{RESULTS}

\section{Demographic characteristics and diagnostic results}

Among 468 subjects who finished diagnostic interview, $44.4 \%$ were boys and $55.6 \%$ were girls. The mean age was 13.3 years $(\mathrm{SD}=1.0)$. Of these, $63(13.5 \%)$ students were classified into patient groups according to DSM-IV-TR, with 405 (86.5\%) students as control groups.

The patient groups were divided into 22 (34.9\%) boys and 41 (65.1\%) girls based on gender. In order of severity, 21 (33.3\%) were mildly depressed patients, 27 (42.9\%) were moderately depressed patients, and 15 (23.8\%) were severely depressed patients in the patient groups.

\section{Comparison of the CDI sum scores}

The CDI scores of total subjects ranged from 0 to 51 , and the mean score was 12.03 ( $\mathrm{SD}=9.95)$. The girls had higher CDI mean scores than boys ( $13.22 \pm 10.74$ vs. $10.53 \pm 8.65, \mathrm{p}=0.003)$. The mean scores of the patient groups were three times higher than the control groups $(27.83 \pm 10.13$ vs. $9.57 \pm 7.32, \mathrm{p}<0.001)$.

\section{Screening of depressive disorders and cut-off scores}

The results of the ROC analyses for the total subjects and separated by gender are presented in Figure 1 and Table 1 . In ROC analysis for the total students, overall detecting power of the CDI scale was high [AUC $=0.93,95 \%$ confidence interval (CI) $0.89-0.96]$. A CDI sum score of 20 (sensitivity $=0.83$, specificity $=0.89$ ) was identified as optimal screening cut-off scores

Table 1. Results of ROC analyses for the complete samples and separated by gender

\begin{tabular}{lccc}
\hline & Complete samples & Girls & Boys \\
\hline Cases (N)/Controls (N) & $63 / 468$ & $41 / 260$ & $22 / 208$ \\
AUC (95\% CI) & $0.93(0.89-0.96)$ & $0.92(0.87-0.97)$ & $0.94(0.90-0.98)$ \\
Optimal cut-off scores & & & $\geq 17$ \\
Cut-off score & $\geq 20$ & $0.86(0.70-0.94)$ & $0.86(0.64-0.96)$ \\
Sensitivity (95\% CI) & $0.83(0.70-0.91)$ & $0.86(0.81-0.90)$ & $0.87(0.81-0.91)$ \\
Specificity (95\% CI) & $0.89(0.86-0.92)$ & & $\geq 20$ \\
Gender-unspecific cut-off score & & $\geq 20$ & $0.77(0.54-0.91)$ \\
Cut-off score & & $0.86(0.70-0.94)$ & $0.93(0.87-0.96)$ \\
Sensitivity (95\% CI) & & $0.86(0.81-0.90)$ & \\
Specificity (95\% CI) & & & \\
\hline
\end{tabular}

AUC: area under the curve, CI: confidence interval, ROC: receiver-operating characteristics 
Table 2. Comparison of CDI sum scores and cut-off scores for rating severity

\begin{tabular}{|c|c|c|c|c|}
\hline & \multirow{2}{*}{ No depression } & \multicolumn{3}{|c|}{ Major depressive disorder } \\
\hline & & Mild & Moderate & Severe \\
\hline Cases $(\mathrm{N}) /$ Controls $(\mathrm{N})$ & $405 / 0$ & $21 / 405$ & $27 / 426$ & $15 / 453$ \\
\hline \multicolumn{5}{|c|}{ Comparison of CDI sum scores and CGI-S scores } \\
\hline CDI mean (SD) & $9.57(7.32)$ & $25.62(11.74)$ & $27.56(8.22)$ & $31.40(10.53)$ \\
\hline CGI-S mean (SD) & $2.10(1.24)$ & $3.19(0.87)$ & $4.11(0.51)$ & $5.47(0.52)$ \\
\hline \multicolumn{5}{|l|}{ Results of ROC analyses } \\
\hline AUC (95\% CI) & & $0.86(0.77-0.95)$ & $0.93(0.89-0.96)$ & $0.92(0.87-0.97)$ \\
\hline \multicolumn{5}{|l|}{ Cut-off score by severity } \\
\hline Cut-off score & & $\geq 15$ & $\geq 20$ & $\geq 25$ \\
\hline Sensitivity (95\% CI) & & $0.86(0.63-0.96)$ & $0.89(0.70-0.97)$ & $0.80(0.51-0.95)$ \\
\hline Specificity $(95 \% \mathrm{CI})$ & & $0.76(0.75-0.83)$ & $0.86(0.82-0.89)$ & $0.90(0.87-0.93)$ \\
\hline
\end{tabular}

AUC: area under the curve, CDI: Children's Depression Inventory, CGI-S: Clinical Global Impression-Severity, CI: confidence interval, SD: standard deviation

$(\mathrm{Y}=0.72) .54 \%$ of those indicated as depressed by a sum score above 20 were also diagnosed with a major depressive disorder according to the K-SADS-PL-K (PPV). $97 \%$ of those with a sum score below 20 did not meet diagnostic criteria either (NPV).

When the students were divided into gender, the optimal cut-off score for girls was 20 (sensitivity $=0.86$, specificity $=0.86$, $\mathrm{PPV}=0.54, \mathrm{NPV}=0.97)$. For boys, the optimal cut-off score was 17 (sensitivity $=0.86$, specificity $=0.87, \mathrm{PPV}=0.43, \mathrm{NPV}=0.98$ ). When the cut-off score 20 was applied for boys, the sensitivity (0.77) of the screening cut-off score was slightly lower than for the gender-specific cut-off score $(\geq 17)$, whereas specificity $(0.93)$ was slightly higher. These slight differences were not significant $(\mathrm{p}=0.5)$.

\section{Rating of depression severity}

After diagnostic interview with K-SADS-PL-K, the patients were selected and classified into three groups as mildly, moderately, and severely depressed patients by depressive symptom severity. The CGI mean scores separated by depression severity were statistically significant $(\mathrm{p}<0.001)$ for showing mildly depressed (3.19 \pm 0.87$)$, moderately depressed $(4.11 \pm 0.51)$, and severely depressed subjects $(5.47 \pm 0.52)$.

ROC analyses identified the cut-off scores according to symptom severity (Table 2): 15 for mildly depressed (sensitivity $=0.86$, specificity $=0.76$ ), 20 for moderately depressed (sensitivity $=0.89$, specificity $=0.86$ ), and 25 for severely depressed subjects (sensitivity $=0.80$, specificity $=0.90$ ).

\section{DISCUSSION}

To our knowledge, this is the first study reporting sensitivity, specificity, and AUC parameters of the CDI cut-off scores in Korean adolescents. The results regarding our two aims sug- gest that: 1) the optimal cut-off score for screening depression was $20 ; 2$ ) according to severity, the cut-off scores were 15 for mildly depressed, 20 for moderately depressed, and 25 for severely depressed subjects. The AUC results demonstrated that the CDI cut-off scores of our study were useful for classifying depressed and non-depressed youth and rating the severity of depressive symptoms.

In this study, a cut-off score of 20 had an NPV of 97\%, suggesting this cut-off score would result in a small number of missed cases. This cut-off score is the same as the originally suggested score by Kovacs, whose study was performed with a sample of 1,226 American public school children aged 7-16 years. ${ }^{11}$ In another study with a clinical sample of 80 European youths, a cut-off score of 20 minimized the proportion of false positives but resulted in a higher proportion of false negatives as in our study. ${ }^{20}$ Thus, this cut-off score is proper in screening because correctly ruling out depression is more important than correctly diagnosing depression in the general population.

There was a gender difference regarding the screening cutoff scores: 20 for girls, 17 for boys. However, it is noteworthy that a score of 20 is an acceptable screening cut-off value for both boys and girls. When we tested the change of detection power between a cut-off score of 17 and 20 in boys, there was no statistical significance of sensitivity and specificity between the two scores. Thus, when clinicians and researchers use the CDI for the purpose of screening depression, a cut-off score of 20 can apply regardless of the clients' gender.

When we examined the optimal cut-off scores in order of depression severity, a cut-off score of 20 has a high specificity sufficient to detect moderate or severe depression. Whereas a cut-off score of 15 is enough to identify depression in even mild episodes, because of the high sensitivity. Kovacs ${ }^{11}$ reported that the cut-off score of 20 was suggested for screening in a 
general population in which the prevalence of depression is likely to be low, and a cut-off score of 13 was suggested for clinic-referred samples in which the prevalence of depression is likely to be higher. Likewise, in our study, we propose that the cut-off score of 20 is optimal for general populations and the cut-off score of 15 is proper for clinical populations.

The mean score of the CDI in our study was higher than in Western studies. ${ }^{9,20,21}$ Korean studies reveal that the mean score of the CDI tends to be higher than that of Western studies. ${ }^{12,22,23}$ Some authors postulated that the false positive rate would increase when the cut-off score which was originally suggested by Kovacs was applied in Korean populations..$^{24}$ For this reason, they suggested $22-25$ as cut-off scores for the CDI. In our study, however, the false positive rate was not higher than in Western studies when applying the cut-off score of $20 .^{20}$ Some methodological considerations arise from this issue. The cut-off value that is estimated from the mean and standard deviation of the studied population has some limitations. Alegret et al. ${ }^{25}$ discussed this issue. They pointed out that the cut-off values using this strategy have the limitation that the classification of "abnormal" depends on the characteristics of the normative sample and does not provide an empirical estimation of the relevant cut-off value for abnormal states. For complementing this issue, we conducted the ROC analyses rather than using a method which depended on deviation from the mean score. ROC analysis has been known to be the best analytic method to validate the predictive power and find the optimal cut-off score of the screening instrument.

Another methodological consideration regarding this issue is the confirmatory diagnostic tool. To set a cut-off score, it is essential to compare the score of the questionnaire with the corresponding diagnosis. We used the K-SADS-PL-K as the gold standard for diagnosing depression. The K-SADS-PL-K is a desirable diagnostic tool with good reliability and validity. ${ }^{16}$ The structured interview with K-SADS-PL-K was able to reduce the false positive and false negative diagnosis of MDD in our study.

The present study has several limitations. First, the participants in our study included students aged 12-16. Thus the result of our study may be inapplicable to screening of depression in other age groups. Because depression is more prevalent in adolescents than children, the CDI cut-off score may be lower in adolescents than children. Further studies will be needed to apply our results to a child population. Second, referral bias should be considered because the adolescents or their caregivers were not randomly selected. It is thus possible that adolescents who have been concerned about their mental health or already have depression may have participated in our study. Such participants may have overstated their symptoms, and diagnosis of depression may therefore have been overestimated. In our study, the overall rate of depression was $13.5 \%$, which is relatively high compared with some other Korean studies. ${ }^{13,14,23}$ However, the validity of diagnosis in our study is supported by structured diagnostic interview, which is more reliable than other questionnaire-based studies. Third, the possibility exists that a high prevalence rate of depression may have interfered with our results (e.g., lower cut-off scores).

Despite these limitations, the strength of our study was in using a systematic approach to set the cut-off scores. Thus, the findings of our study could be a helpful guideline for screening depression and rating severity in adolescents. Although diagnosis of depression should be confirmed through the multiple assessments, the cut-off score of our study will provide a quick and tentative threshold for classifying depression.

\section{Acknowledgments}

This work was supported by the Dong-A University research fund.

\section{REFERENCES}

1. Kessler RC, Avenevoli S, Costello EJ, Green JG, Gruber MJ, Heeringa S, et al. National comorbidity survey replication adolescent supplement (NCS-A): II. Overview and design. J Am Acad Child Adolesc Psychiatry 2009;48:380-385.

2. Wade TJ, Cairney J, Pevalin DJ. Emergence of gender differences in depression during adolescence: national panel results from three countries. J Am Acad Child Adolesc Psychiatry 2002;41:190-198.

3. Biederman J, Faraone S, Mick E, Lelon E. Psychiatric comorbidity among referred juveniles with major depression: fact or artifact? J Am Acad Child Adolesc Psychiatry 1995;34:579-590.

4. Birmaher B, Arbelaez C, Brent D. Course and outcome of child and adolescent major depressive disorder. Child Adolesc Psychiatr Clin N Am 2002;11:619-637.

5. Lewinsohn PM, Rohde P, Seeley JR. Major depressive disorder in older adolescents: prevalence, risk factors, and clinical implications. Clin Psychol Rev 1998;18:765-794.

6. Kovacs M. Presentation and course of major depressive disorder during childhood and later years of the life span. J Am Acad Child Adolesc Psychiatry 1996;35:705-715.

7. Kovacs M. The Children's Depression Inventory (CDI). Psychopharmacol Bull 1985;21:995-998.

8. Smucker MR, Craighead WE, Craighead LW, Green BJ. Normative and reliability data for the Children's Depression Inventory. J Abnorm Child Psychol 1986;14:25-39.

9. Stark KD, Laurent J. Joint factor analysis of the Children's Depression Inventory and the Revised Children's Manifest Anxiety Scale. J Clin Child Psychol 2001;30:552-567.

10. Chareeb G, Beshai J. Arabic version of the Children's Depression Inventory: reliability and validity. J Clin Child Psychol 1989;18:323-326.

11. Kovacs M. Children's Depression Inventory (CDI) Manual. New York: Multi-Health Systems; 1992.

12. Cho SC, Lee YS. Development of the Korean form of the Kovacs' Childeren's Depression Inventory. J Korean Neuropsychiatr Assoc 1990;29: 943-956.

13. Yang JW, Kim YJ, Kim HS, Shin KM, Shin YM. Difference between children's self-reports on depression and parents' assessment of children's behaviors. J Korean Acad Child and Adolesc Psychiatry 2012;23: 76-81.

14. Choi HW, Lee MS, Lim MH, Kwon HJ, Ha M, Yoo SJ, et al. The prevalence and epidemiological characteristics of childhood depressive disorder in South Korea: self reported study. J Korean Acad Child Adolesc Psychiatry 2012;23:134-142. 
15. American Psychiatry Association. Diagnostic and Statistical Manual of Mental Disorders, Fourth Edition: DSM-IV-TR. Washington DC: American Psychiatric Association; 2000.

16. Kim YS, Cheon KA, Kim BN, Chang SA, Yoo HJ, Kim JW, et al. The reliability and validity of Kiddie-Schedule for Affective Disorders and Schizophrenia-Present and Lifetime Version- Korean version (K-SADSPL-K). Yonsei Med J 2004;45:81-89.

17. Berk M, Ng F, Dodd S, Callaly T, Campbell S, Bernardo M, et al. The validity of the CGI severity and improvement scales as measures of clinical effectiveness suitable for routine clinical use. J Eval Clin Pract 2008;14:979-983.

18. Metz CE. Basic principles of ROC analysis. Semin Nucl Med 1978;8: 283-298.

19. Youden WJ. Index for rating diagnostic tests. Cancer 1950;3:32-35.

20. Timbremont B, Braet C, Dreessen L. Assessing depression in youth: relation between the Children's Depression Inventory and a structured interview. J Clin Child Adolesc Psychol 2004;33:149-157.
21. Sorensen MJ, Frydenberg M, Thastum M, Thomsen PH. The Children's Depression Inventory and classification of major depressive disorder: validity and reliability of the Danish version. Eur Child Adolesc Psychiatry 2005;14:328-334.

22. Yang JW, Kim YJ, Kim HS, Shin KM, Shin YM. Difference between children's self-reports on depression and parents' assessment of children's behaviors. J Korean Acad Child Adolesc Psychiatry 2012;23:7681.

23. Park JI. Factor analysis of Children's Depression Inventory. J Korean Neuropsychiatr Assoc 2008;47:555-560.

24. Shin MS, Kim MK. Asessment of childhood depression. J Child Adolesc Psychiatry 1994;5:12-27.

25. Alegret M, Espinosa A, Valero S, Vinyes-Junque G, Ruiz A, Hernandez I, et al. Cut-off Scores of a Brief Neuropsychological Battery (NBACE) for Spanish individual adults older than 44 Years old. PLoS One 2013;8: e76436. 